\title{
Study of Omentin-1 and Chemerin as Predictors of Coronary Artery Disease in Elderly Patients, with Retrospective Studying of their Relation to Diabetes Mellitus Type 2
}

Maher Borai Mohammad*1, Mohammad Ibrahim Amin², Ahmad Soliman Sallam¹, Abeer Abdalla Fekry ${ }^{1}$ Departments of ${ }^{1}$ Clinical Pathology and ${ }^{2}$ Cardiology, Faculty of Medicine, Zagazig University, Egypt.

*Corresponding author: Maher Borai Mohammad, Mobile: (+20) 01116441130, E-Mail: maherborai@ yahoo.com

\section{ABSTRACT}

Introduction: Omentin-1, is an adipokine, inhibits TNF-induced inflammation of vascular smooth muscle cells, so its decreased levels in plasma of patients with coronary artery disease (CAD), indicated that omentin-1 may also be involved in the occurrence of CAD.

Objective: Study the alternation of plasma levels of omentin-1 and chemerin in patients with coronary artery diseases. Patients and methods: This case-control study was carried out on 120 patients with coronary artery disease and 50 healthy volunteers, in the period from January 2018 to June 2019, divided into control group: 50 healthy volunteers, stable angina pectoris group: 60 cases, acute coronary syndrome (ACS) group: 60 cases, which were subclassified into; (a) 20 unstable angina (USA) cases; (b) 20 segment elevation myocardial infarction (STEMI) cases; (c) 20 non ST segment elevation myocardial infarction (NSTEMI) cases.

Results: There was a highly significant difference between the studied groups in hsCRP, omentin- 1 and chemerin levels with significant difference in SA group and ACS groups compared to control group. Regarding acute coronary syndrome (ACS) groups, there was significant difference in hsCRP between USA and infarction groups. Chemerin showed significant difference between USA and STEMI and USA and Non STE MI. While omentin-1 showed no significant difference between ACS subgroups. Regarding CAD without DM type2 and CAD with DM type2. There was high statistical significant difference between the groups as regarding FBG, HbA1c, total cholesterol (T. Chol), HDL-C, LDL-C, hsCRP, chemerin and omentin-1.

Conclusion: increasing total cholesterol, triglycerides, LDL-C, high- sensitivity C- reactive protein (hsCRP) and chemerin. Decreasing HDL-C and Omentin- 1 are independent predictors of CAD in type 2 diabetic patients.

Keywords: Chemerin, Coronary Artery Disease, Diabetes Mellitus type 2, Omentin-1.

\section{INTRODUCTION}

Cardiovascular disease (CVD) represents the leading cause of mortality in developed countries. Controlling the risk factors such as lifestyle modification, glycemic control, blood pressure (BP), and body mass index (BMI) can achieve great reduction in mortality (1). Diabetes, because of its vascular complications that is associated with the hyperglycemia-induced oxidative stress, lack of endothelial regeneration and endothelial dysfunction results in abnormal vascular remodeling and impaired neovascularization ${ }^{(2)}$.

The adipokine theory is a widely accepted theory. It suggests that adipocytokines are involved in the inflammatory response, which directly promotes the occurrence of $\mathrm{CAD}^{(3,4)}$.

Omentin-1, is an adipokine, increases sensitivity to insulin, its decreased levels may be responsible for impaired glucose homeostasis. Omentin-1 inhibits TNF-induced inflammation of vascular smooth muscle cells, so its decreased levels in plasma of patients with CAD, indicated that omentin-1 may also be involved in the occurrence of $\operatorname{ACS}{ }^{(5,6)}$.

Chemerin has been also hypothesized as a possible link between obesity and the development of diabetes mellitus type 2 (DM2). Chemerin by its chemoattractant functions and receptors expressed by tissue macrophages induces leukocyte migration, functions in the development of inflammatory diseases, including atherosclerosis ${ }^{(7)}$.

The aim of the present work was to study the alternation of plasma levels of omentin- 1 and chemerin in patients with coronary artery diseases, its relation to DM2 and its availability to be used as predictors for CAD in elderly patients.

\section{PATIENTS AND METHODS}

This case-control study was carried out on 120 patients with coronary artery disease, referred to Cardiology and Clinical Pathology Departments, Zagazig University Hospitals, and 50 healthy volunteers matched for age and sex from the general population in the period from January 2019 to June 2020.

Participants enrolled in this study were divided into three groups according to disease state regarding CAD that was diagnosed by ECG changes, cardiac enzymes and coronary angiography that was performed under local anesthesia using Seldinger technique with right femoral approach ${ }^{(8)}$. Gensini score was calculated from the number of stenotic coronary artery segments and the degree of lumen stenosis $^{(9)}$. Participants were divided into:

Control group: 50 volunteers from the general population matched for age and sex without evidence 
of any coronary artery disease neither by history nor by investigations.

Stable angina pectoris group: 60 cases diagnosed as typical chest pain at predicted level of exertion plus stress test of ECG and Echo.

Acute coronary syndrome Group: 60 cases which were subclassified into: (a) 20 unstable angina cases diagnosed as angina at low effort or changes in severity and duration or angina with first negative cardiac enzymes. (b) 20 ST segment elevation myocardial infarction (STEMI) cases. (c) 20 non ST segment elevation myocardial infarction (NSTEMI) cases.

Groups $b$ and $c$ were diagnosed by performing ECG to identify evolving damage of heart muscle, if ECG shows elevation of ST segment, which in the context of severe typical chest pain is strongly indicative of an acute MI. In the absence of ST segment elevation heart damage is detected by cardiac markers, if there is evidence of damage, chest pain is attributed to non-STMI.

The stable angina pectoris and acute coronary syndrome groups were reclassified according to presence of type-2 diabetes (which was defined according to American Diabetes Association ${ }^{(10)}$ into: Group CAD+DM: including 75 coronary patients, which are diabetics. Group CAD without DM: including 45 coronary patients, which are nondiabetics.

\section{Ethical approval:}

An approval of the study was obtained from Zagazig University academic and ethical committee. An informed consent was taken from all participants in the research and the privacy of the data was greatly considered.

Inclusion criteria: Stable angina pectoris. Unstable angina pectoris. Myocardial infarction is ST segment elevation (STEMI) and non ST segment elevation (non STEMI). Subjects of 60 years old or more according WHO definition of elderly among Egyptian (Dar el salaam MDS meeting 2001).

Exclusion criteria: Severe liver/kidney dysfunction; severe systemic disease; malignancy; infectious diseases; autoimmune disease or connective tissue disease; and a major trauma or surgical operation; patients less than 60 years.

All candidates of the study were subjected to the following:

(1) Full history taking

(2) Thorough physical examination

(3) Laboratory investigations:

a) Complete Blood Count (CBC) by (Sysmex-Kx$21 \mathrm{~N})$.

b) Random serum glucose, AST, ALT, B1. Urea, creatinine, TC, TG, HDL-C by (Cobas Hitachi C501, Roche Germany). c) High sensitive C-Reactive protein by (Sigbeckman Coulten AU480).

d) $\mathrm{HbAlc}$ was determined by competitive turbidimetric inhibition immunoassay on the Cobas 6000 auto-analyzer (Roche Diagnostics).

e) Determination of serum Omentin-1 level: using ELISA kit (WKEA MED SUPPLIES CO. China) with detection range of $50 \mathrm{ng} / \mathrm{L}$ to 1000 $\mathrm{ng} / \mathrm{L}$.

f) Determination of serum chemerin levels: were determined using a commercially available enzyme-linked immunosorbent assay (ELISA) kit (Glory Science Co, USA), according to manufacturer's protocol. The detection limit is $20-1200 \mathrm{ng} / \mathrm{L}$.

\section{Principle of the assay:}

The kits assay human omentin-1 or chemerin levels in the sample, using purified human omentin1 or chemerin antibodies to coat microtiter plate well, make solid-phase antibody, then either omentin-1 or chemerin was added to wells. Combined antigen, a second antibody, which is HRP labeled then added, to form antibody-antigen-antibody enzyme labeled complex. Finally the color change was measured spectrophotometrically at a wavelength of $450 \mathrm{~nm}$. The concentrations were then determined by standard curve.

\section{Sample processing:}

Venous blood was collected into: EDTA anticoagulant tube, for CBC. Plain tubes, to collect serum. One tube was centrifuged to separate serum, kept at $-70^{\circ}$ for determination of omentin- 1 and chemerin. While the second for other tests.

\section{Statistical analysis}

The collected data were coded, processed and analyzed using the SPSS (Statistical Package for Social Sciences) version 22 for Windows ${ }^{\circledR}$ (IBM SPSS Inc, Chicago, IL, USA). Data were tested for normal distribution using the Shapiro Walk test. Qualitative data were represented as frequencies and relative percentages. Chi square test $(\chi 2)$ to calculate difference between two or more groups of qualitative variables. Quantitative data were expressed as mean \pm SD (Standard deviation). Independent samples t-test was used to compare between two independent groups of normally distributed variables (parametric data). $\mathrm{P}$ value $<0.05$ was considered significant.

\section{RESULTS}

Body mass index (BMI) showed significant difference between control group and both the SA, ACS groups. There was high statistical significant difference between groups as regard occurrence of diabetes mellitus and hypertension, total cholesterol ( $\mathrm{T} \mathrm{Chol}$ ) and LDL-C, with more increase in SA and ACS groups. Meanwhile, there was significant difference between control and SA and control and ACS for Trg. and significant difference between control and ACS and 
between SA and ACS groups as regard HDL-C. As regard hsCRP there was a significant difference between control and other groups and in between SA and ACS groups. Omentin-1 level showed statistically significant decrease in its level in SA group and ACS groups compared to control group.
Chemerin level showed significant increase in its level in SA group and ACS groups compared to control group, there was also significant increase between SA and ACS groups (Table 1).

Table (1): Studying of demographic, clinical and Laboratorial data among the studied groups

\begin{tabular}{|c|c|c|c|c|c|}
\hline & $\begin{array}{c}\text { Control group } \\
(\mathrm{n}=50)\end{array}$ & $\begin{array}{c}\mathrm{SA} \\
(\mathrm{n}=60)\end{array}$ & $\begin{array}{c}\mathrm{ACS} \\
(\mathrm{n}=60)\end{array}$ & $\mathbf{P}$ & LSD \\
\hline $\begin{array}{l}\text { Age: (years) } \\
X \pm S D\end{array}$ & $64.9 \pm 6.07$ & $63.5 \pm 4.84$ & $64.4 \pm 5.41$ & 0.387 & \\
\hline $\begin{array}{l}\text { BMI:(Kg/M 2) } \\
X \pm S D\end{array}$ & $21.5 \pm 1.79$ & $23.2 \pm 3.31$ & $23.6 \pm 3.93$ & 0.002 & $\begin{array}{l}<\mathbf{0 . 0 5} \text { P1 } \\
<\mathbf{0 . 0 5} \text { P2 } \\
>0.05 \text { P3 }\end{array}$ \\
\hline $\begin{array}{l}\text { Sex: } \mathbf{N o}(\%) \\
\text { Female } \\
\text { Male }\end{array}$ & $\begin{array}{l}25(50 \%) \\
25(50 \%)\end{array}$ & $\begin{array}{l}14(23.3 \%) \\
46(76.7 \%)\end{array}$ & $\begin{array}{l}22(36.7 \%) \\
38(63.3 \%)\end{array}$ & 0.015 & \\
\hline $\begin{array}{l}\text { Diabetes M: No (\%) } \\
\text { Yes } \\
\text { No }\end{array}$ & $\begin{array}{c}0(0 \%) \\
50(100 \%)\end{array}$ & $\begin{array}{l}30(50 \%) \\
30(50 \%)\end{array}$ & $\begin{array}{l}45(75 \%) \\
15(25 \%)\end{array}$ & 0.001 & \\
\hline $\begin{array}{l}\text { Hypertension: } N o(\%) \\
Y e S \\
N o\end{array}$ & $\begin{array}{c}0(0 \%) \\
50(100 \%)\end{array}$ & $\begin{array}{l}40(66.7 \%) \\
20(33.3 \%)\end{array}$ & $\begin{array}{l}40(66.7 \%) \\
20(33.3 \%)\end{array}$ & 0.001 & \\
\hline $\begin{array}{l}\text { T. Chol (mg/ dl) } \\
\mathrm{X} \pm \mathrm{SD}\end{array}$ & $131.7 \pm 6.6$ & $157.4 \pm 6.8$ & $222.1 \pm 7.2$ & $<0.001$ & $\begin{array}{l}<0.05 \text { P1 } \\
<0.05 \text { P2 } \\
<0.05 \text { P3 }\end{array}$ \\
\hline $\begin{array}{l}\text { Trg. }(\boldsymbol{m g} / \mathbf{d l}) \\
X \pm S D\end{array}$ & $71.1 \pm 2.2$ & $55 \pm 6.2$ & $61.5 \pm 9.2$ & 0.005 & $\begin{array}{l}<\mathbf{0 . 0 5} \text { P1 } \\
<0.05 \text { P2 } \\
>0.05 \text { P3 }\end{array}$ \\
\hline $\begin{array}{l}\text { HDL-C: }(\boldsymbol{m g} / \boldsymbol{d l}) \\
X \pm S D\end{array}$ & $42.8 \pm 1.7$ & $35.4 \pm 3.3$ & $28.6 \pm 7.5$ & 0.007 & $\begin{array}{l}>0.05 \text { P1 } \\
<0.05 \text { P2 } \\
<0.05 \text { P3 }\end{array}$ \\
\hline $\begin{array}{l}\text { LDL-C: }(\boldsymbol{m g} / \boldsymbol{d l}) \\
X \pm S D\end{array}$ & $111.6 \pm 17.3$ & $102.5 \pm 23.9$ & $144.3 \pm 7.6$ & $<0.001$ & $\begin{array}{l}>0.05 \text { P1 } \\
<\mathbf{0 . 0 5} \text { P2 } \\
<\mathbf{0 . 0 5} \text { P3 }\end{array}$ \\
\hline $\begin{array}{l}\text { ALT: }(\boldsymbol{U} / \mathbf{L}) \\
X \pm S D\end{array}$ & $20.6 \pm 2.2$ & $15.7 \pm 2.4$ & $34.3 \pm 7.09$ & 0.114 & \\
\hline $\begin{array}{l}\text { AST: }(\boldsymbol{U} / \boldsymbol{L}) \\
X \pm S D\end{array}$ & $21.9 \pm 1.1$ & $16.6 \pm 3.4$ & $46.8 \pm 2.2$ & 0.001 & \\
\hline $\begin{array}{l}\text { Urea: }(\boldsymbol{m g} / \mathrm{dl}) \\
X \pm S D\end{array}$ & $18.2 \pm 4.6$ & $21.2 \pm 3.5$ & $20.9 \pm 4.2$ & 0.236 & \\
\hline $\begin{array}{l}\text { Creat : }\left(\begin{array}{ll}m g & d l\end{array}\right) \\
X \pm S D\end{array}$ & $0.88 \pm 0.14$ & $0.92 \pm 0.25$ & $0.98 \pm 0.37$ & 0.464 & \\
\hline $\begin{array}{l}\text { hsCRP: }(\mathbf{m g} / \mathbf{L}) \\
X \pm S D\end{array}$ & $0.62 \pm 0.1$ & $2.6 \pm 0.1$ & $3.4 \pm 0.2$ & $<0.001$ & $\begin{array}{l}<0.05 \mathrm{P} 1 \\
<0.05 \mathrm{P} 2 \\
<0.05 \mathrm{P} 3\end{array}$ \\
\hline $\begin{array}{l}\text { Omentin-1:(ng/ L) } \\
X \pm S D\end{array}$ & $136.7 \pm 3.16$ & $118.9 \pm 3.01$ & $115.7 \pm 19.2$ & 0.01 & $\begin{array}{l}<0.05 \mathrm{P} 1 \\
<0.05 \mathrm{P} 2 \\
>0.05 \mathrm{P} 3\end{array}$ \\
\hline $\begin{array}{l}\text { Chemerin: (ng/L) } \\
X \pm S D\end{array}$ & $148 \pm 5.3$ & $574 \pm 6.2$ & $671 \pm 4.2$ & $<0.001$ & $\begin{array}{l}<0.05 \mathrm{P} 1 \\
<0.05 \mathrm{P} 2 \\
<0.05 \mathrm{P} 3\end{array}$ \\
\hline
\end{tabular}

LSD: Least significant post hoc test P1: control vs SA P2: control vs ACS. P3: SA vs ACS.

Regarding ACS groups, hsCRP showed that there was significant difference between USA and infarction groups. Chemerin showed significant difference between USA and STE MI and USA and Non STE (Table 2). 
Table (2): Study of demographic, clinical and Laboratorial data among ACS groups

\begin{tabular}{|c|c|c|c|c|c|}
\hline & $\begin{array}{c}\text { US angina } \\
(n=10)\end{array}$ & $\begin{array}{c}\text { STE MI } \\
(n=10)\end{array}$ & $\begin{array}{c}\text { Non STEMI } \\
(\mathbf{n}=10)\end{array}$ & $\mathbf{P}$ & LSD \\
\hline $\begin{array}{l}\text { Age: (years) } \\
X \pm S D \\
\text { Range }\end{array}$ & $\begin{array}{c}66.9 \pm 7.78 \\
60-81\end{array}$ & $\begin{array}{c}62.8 \pm 3.79 \\
60-73\end{array}$ & $\begin{array}{c}63.5 \pm 2.95 \\
60-70\end{array}$ & 0.197 & \\
\hline $\begin{array}{l}\text { BMI:(Kg/M 2) } \\
X \pm S D\end{array}$ & $24.1 \pm 4.09$ & $23.6 \pm 3.92$ & $23.1 \pm 4.15$ & 0.877 & \\
\hline $\begin{array}{l}\text { Sex: No, }(\%) \\
\text { Female } \\
\text { Male }\end{array}$ & $\begin{array}{l}12(60) \\
8(40)\end{array}$ & $\begin{array}{l}2(10) \\
18(90)\end{array}$ & $\begin{array}{c}8(40) \\
12(60)\end{array}$ & $<0.005$ & \\
\hline $\begin{array}{l}\text { DM: No, }(\%) \\
Y e s \\
\text { No }\end{array}$ & $\begin{array}{l}14(70) \\
6(30)\end{array}$ & $\begin{array}{l}16(80) \\
4(20)\end{array}$ & $\begin{array}{l}14(70) \\
6(30)\end{array}$ & 0.771 & \\
\hline $\begin{array}{l}\text { Hypertension: No, }(\%) \\
\text { Yes } \\
\text { No }\end{array}$ & $\begin{array}{l}14(70) \\
6(30)\end{array}$ & $\begin{array}{l}12(60) \\
8(40)\end{array}$ & $\begin{array}{l}14(70) \\
6(30)\end{array}$ & 0.741 & \\
\hline $\begin{array}{l}\text { T. Chol: }(\mathbf{m g} / \mathbf{d l}) \\
X \pm \text { SD }\end{array}$ & $238 \pm 22.8$ & $225.8 \pm 26.5$ & $202.7 \pm 28.1$ & 0.566 & $\begin{array}{l}>0.05 \mathrm{P} 1 \\
>0.05 \mathrm{P} 2 \\
>0.05 \mathrm{P} 3\end{array}$ \\
\hline $\begin{array}{l}\text { Trg. }(m g / d l) \\
X \pm S D\end{array}$ & $57.9 \pm 11.9$ & $66.3 \pm 3.8$ & $60.5 \pm 7.4$ & 0.623 & $\begin{array}{l}>0.05 \mathrm{P} 1 \\
>0.05 \mathrm{P} 2 \\
>0.05 \mathrm{P} 3\end{array}$ \\
\hline $\begin{array}{l}\text { HDL-C: }(\boldsymbol{m g} / \boldsymbol{d l}) \\
X \pm S D\end{array}$ & $35 \pm 2.6$ & $39 \pm 1.5$ & $36 \pm 1.9$ & 0.731 & $\begin{array}{l}>0.05 \mathrm{P} 1 \\
>0.05 \mathrm{P} 2 \\
>0.05 \mathrm{P} 3\end{array}$ \\
\hline $\begin{array}{l}\text { LDL-C: }(\boldsymbol{m g} / \boldsymbol{d l}) \\
X \pm S D\end{array}$ & $154.7 \pm 9.8$ & $146.7 \pm 17.2$ & $131.7 \pm 18.2$ & 0.560 & $\begin{array}{l}>0.05 \mathrm{P} 1 \\
>0.05 \mathrm{P} 2 \\
>0.05 \mathrm{P} 3\end{array}$ \\
\hline $\begin{array}{l}\text { ALT: }(\boldsymbol{U} / L) \\
X \pm S D\end{array}$ & $16.4 \pm 3.5$ & $23.5 \pm 4.5$ & $33 \pm 4.9$ & 0.344 & \\
\hline $\begin{array}{l}\text { AST: }(\boldsymbol{U} / \boldsymbol{L}) \\
X \pm S D\end{array}$ & $28 \pm 5.8$ & $51.8 \pm 9.2$ & $70.6 \pm 7.3$ & 0.392 & \\
\hline $\begin{array}{l}\text { Urea: }(\boldsymbol{m g} / \mathrm{dl}) \\
X \pm S D\end{array}$ & $23.8 \pm 1.4$ & $17.7 \pm 4.8$ & $21.2 \pm 5.24$ & 0.265 & \\
\hline $\begin{array}{l}\text { Creat : }\left(\begin{array}{ll}m g & d l\end{array}\right) \\
X \pm S D\end{array}$ & $1.11 \pm 0.2$ & $0.9 \pm 0.06$ & $0.8 \pm 0.03$ & 0.259 & \\
\hline $\begin{array}{l}\text { hsCRP: }(\mathbf{m g} / \mathbf{L}) \\
X \pm S D\end{array}$ & $2.61 \pm 0.5$ & $3.7 \pm 0.34$ & $3.8 \pm 0.46$ & $<0.001 *$ & $\begin{array}{l}<0.05 \mathrm{P} 1 \\
<0.05 \mathrm{P} 2 \\
>0.05 \mathrm{P} 3\end{array}$ \\
\hline $\begin{array}{l}\text { Omentin-1:(ng/ L) } \\
X \pm S D\end{array}$ & $109.6 \pm 15.2$ & $125.7 \pm 14.5$ & $111.8 \pm 23.9$ & 0.015 & \\
\hline $\begin{array}{l}\text { Chemerin }(\mathbf{n g} / \mathbf{L}) \\
X \pm S D\end{array}$ & $634 \pm 18.2$ & $668 \pm 31.3$ & $671 \pm 25.4$ & $<0.001$ & $\begin{array}{l}<0.05 \mathrm{P} 1 \\
<0.05 \mathrm{P} 2 \\
>0.05 \mathrm{P} 3\end{array}$ \\
\hline
\end{tabular}

LSD: Least significant post hoc test P1: control vs SA P2: control vs ACS. P3: SA vs ACS.

On studying the coronary artery disease participants on basis of diabetic parameters, we divided them into: CAD without DM type 2 and CAD with DM type 2 . There was high statistical significant difference between the groups as regarding FBG, HbA1c, T Chol., HDL-C, LDL-C, hsCRP, chemerin and omentin-1 (Table 3). 
Table (3): Study of patients according to presence or absence of diabetes mellitus with CAD

\begin{tabular}{|l|c|c|c|c|c|}
\hline & $\begin{array}{c}\text { Control } \\
\text { group } \\
(\mathrm{N}=50)\end{array}$ & $\begin{array}{c}\text { CAD without } \\
\text { DM type 2 } \\
(\mathrm{N}=45)\end{array}$ & $\begin{array}{c}\text { CAD with } \\
\text { DM type 2 } \\
(\mathrm{N}=75)\end{array}$ & p-value & LSD \\
\hline $\begin{array}{l}\text { FBG (mg/dl) } \\
X \pm S D\end{array}$ & $79 \pm 4.3$ & $82 \pm 3.2$ & $173 \pm 23.2$ & $<0.001$ & $\begin{array}{l}\mathrm{P} 1>0.05 \\
\mathrm{P} 2<0.05 \\
\mathrm{P} 3<0.05\end{array}$ \\
\hline $\begin{array}{l}\text { HbA1c (\%) } \\
X \pm S D\end{array}$ & $3.9 \pm 0.77$ & $4.0 \pm 0.5$ & $5.4 \pm 0.98$ & $<0.001$ & $\begin{array}{l}\mathrm{P} 1>0.05 \\
\mathrm{P} 2<0.05 \\
\mathrm{P} 3<0.05\end{array}$ \\
\hline $\begin{array}{l}\text { T. Chol. (mg/d) } \\
X \pm S D\end{array}$ & $131.7 \pm 26.6$ & $198 \pm 21.5$ & $212 \pm 34.5$ & $<0.001$ & $\begin{array}{l}\mathrm{P} 1>0.05 \\
\mathrm{P} 2<0.05 \\
\mathrm{P} 3<0.05\end{array}$ \\
\hline $\begin{array}{l}\text { Trig. (mg/d) } \\
X \pm S D\end{array}$ & $71.1 \pm 20.2$ & $86 \pm 7.3$ & $89 \pm 8.4$ & $<0.05$ & $\begin{array}{l}\mathrm{P} 1>0.05 \\
\mathrm{P} 2<0.05 \\
\mathrm{P} 3>0.05\end{array}$ \\
\hline $\begin{array}{l}\text { HDL-C (mg/dl) } \\
X \pm S D\end{array}$ & $42.8 \pm 1.7$ & $34 \pm 4.7$ & $31 \pm 3.6$ & $<0.001$ & $\begin{array}{l}\mathrm{P} 1<0.05 \\
\mathrm{P} 2<0.05 \\
\mathrm{P} 3>0.05\end{array}$ \\
\hline $\begin{array}{l}\text { LDL-C (mg/d) } \\
X \pm S D\end{array}$ & $111.6 \pm 17.3$ & $143.7 \pm 15$ & $154 \pm 8.8$ & $<0.001$ & $\begin{array}{l}\mathrm{P} 1<0.05 \\
\mathrm{P} 2<0.05 \\
\mathrm{P} 3<0.05\end{array}$ \\
\hline $\begin{array}{l}\text { hsCRP (mg/L) } \\
X \pm S D\end{array}$ & $0.62 \pm 0.01$ & $2.1 \pm 0.41$ & $4.4 \pm 0.55$ & $<0.001$ & $\begin{array}{l}\mathrm{P} 1<0.05 \\
\mathrm{P} 2<0.05 \\
\mathrm{P} 3<0.05\end{array}$ \\
\hline $\begin{array}{l}\text { Chemerin(ng/ml) } \\
X \pm S D\end{array}$ & $148 \pm 25.3$ & $165 \pm 35$ & $656 \pm 39.2$ & $<0.001$ & $\begin{array}{l}\mathrm{P} 1<0.05 \\
\mathrm{P} 2<0.05 \\
\mathrm{P} 3<0.05\end{array}$ \\
\hline $\begin{array}{l}\text { Omentin-1 (ng/ml) } \\
X \pm S D\end{array}$ & $136.7 \pm 3.16$ & $122.4 \pm 9.5$ & $110.7 \pm 14.2$ & $<0.001$ & $\begin{array}{l}\mathrm{P} 1<0.05 \\
\mathrm{P} 2<0.05 \\
\mathrm{P} 3<0.05\end{array}$ \\
\hline
\end{tabular}

LSD: Least significant post hoc test P1: control vs SA P2: control vs ACS. P3: SA vs ACS.

Logistic regression analysis revealed that increasing total cholesterol, triglycerides, LDL-C, hsCRP and chemerin and decreasing HDL-C and omentin- 1 are independent predictors of CAD in type 2 diabetic patients (Table 4 ).

Table (4) Logistic regression of potential predictors of CAD in type 2 diabetic patients:

\begin{tabular}{|l|c|c|c|c|c|}
\hline & $\boldsymbol{\beta}$ & SE & p-value & OR & 95\% CI \\
\hline BMI (Kg/m $\mathbf{2})$ & +0.034 & 0.076 & 0.660 & 1.034 & $(0.890-1.201)$ \\
\hline FBG (mg/dl) & +0.002 & 0.004 & 0.602 & 1.002 & $(0.994-1.010)$ \\
\hline HbA1c (\%) \# & +0.120 & 0.246 & 0.625 & 1.128 & $(0.697-1.825)$ \\
\hline T. Chol. (mg/dl) & +0.030 & 0.008 & $<\mathbf{0 . 0 0 1}$ & 1.031 & $(1.016-1.046)$ \\
\hline Trig. (mg/dl) & +0.39 & 0.009 & $<\mathbf{0 . 0 0 1}$ & 1.040 & $(1.021-1.059)$ \\
\hline HDL-C (mg/dl) & -0.147 & 0.047 & $\mathbf{0 . 0 0 2}$ & 0.864 & $(0.788-0.946)$ \\
\hline LDL-C (mg/dl) & +0.037 & 0.009 & $<\mathbf{0 . 0 0 1}$ & 1.038 & $(1.019-1.057)$ \\
\hline hsCRP (mg/L) & +1.186 & 0.336 & $<\mathbf{0 . 0 0 1}$ & 3.275 & $(1.695-6.328)$ \\
\hline Chemerin (ng/ml) & +0.014 & 0.004 & $<\mathbf{0 . 0 0 1}$ & 1.014 & $(1.007-1.022)$ \\
\hline Omentin-1(ng/ml) & -0.016 & 0.003 & $<0.001$ & 0.984 & $(0.979-0.990)$ \\
\hline
\end{tabular}

$\boldsymbol{\beta}$ : regression Coefficient. SE: standard error.

OR: Odds Ratio. $95 \%$

CI: $95 \%$ confidence interval. 
Table (5): hsCRP, chemerin and Omentin-1 as a diagnostic markers for CAD; ROC curve Analysis:

\begin{tabular}{|l|c|c|c|c|c|c|}
\hline Cut-off values & SN \% & SP \% & PV \% & NPV \% & Accuracy & AUC \\
\hline $\begin{array}{l}\text { hsCRP } \\
>3.0 \mathrm{mg} / \mathrm{L}\end{array}$ & $91 \%$ & $90 \%$ & $89 \%$ & $90 \%$ & $88 \%$ & 0.946 \\
\hline $\begin{array}{l}\text { Chemerin } \\
>375 \mathrm{ng} / \mathrm{ml}\end{array}$ & $96.7 \%$ & $86.6 \%$ & $88.2 \%$ & $96.3 \%$ & $91.7 \%$ & 0.949 \\
\hline $\begin{array}{l}\text { Omentin } \\
\leq 97.5\end{array}$ & $83.3 \%$ & $71.7 \%$ & $59.5 \%$ & $89.6 \%$ & $75.6 \%$ & 0.816 \\
\hline $\begin{array}{l}\text { Chemerin+Omentin } \\
\text { hsCRP }\end{array}$ & $100 \%$ & $90 \%$ & $90.9 \%$ & $100 \%$ & $95 \%$ & 0.988 \\
\hline
\end{tabular}

SN: Sensitivity. $\quad$ SP: Specificity.

NPV: Negative Predictive Value

95\% CI: 95\% Confidence Interval.

\section{DISCUSSION}

The adipokine theory is a widely accepted theory. It suggests that adipocytokines are involved in the inflammatory response, which directly promotes the occurrence of CAD. Adipose tissue secretes many adipokines including adiponectin, chemerin, omentin-1, leptin, resistin, retinol binding protein 4 , tumor necrosis factor- $\alpha$ (TNF- $\alpha$ ), and interleukin- 6 (IL-6) $^{(3,11-13)}$.

Omentin-1 (Intelectin-1), is an adipokine, increases sensitivity to insulin, its decreased levels may be responsible for impaired glucose homeostasis especially in obese patients ${ }^{(4)}$. Omentin-1 inhibits TNF-induced inflammation of vascular smooth muscle cells (SMCs), so its decreased levels in plasma of patients with CHD, indicated that omentin-1 may also be involved in the occurrence of ACS ${ }^{(5)}$.

Chemerin has increasing evidence for its roles in adipogenesis, energy metabolism. Chemerin has been also hypothesized as a possible link between obesity and the development of diabetes mellitus type 2 (DM2) ${ }^{(7)}$. Chemerin by its chemoattractant functions, receptors expressed by tissue macrophages, induces leukocyte migration, functions in the development of inflammatory diseases, including atherosclerosis ${ }^{\mathbf{1 4}, 15)}$.

Our study showed that there were no statistically significant differences between the studied groups regarding age and sex, but regarding BMI there was significant difference between control and patients. In the contrary to our study, Zhong et al. ${ }^{(16)}$, showed that there were no differences in age, BMI and sex between patients and controls ${ }^{(\mathbf{1 7})}$.

Moreover, as regarding diabetes mellitus and hypertension, in our study there were highly significant differences between studied groups. While, there were no significant difference among ACS subgroups for both. Wang et al. ${ }^{(18)}$, observed a significant correlation between lower omentin-1 levels and higher systolic pressure (SBP). After reviewing several large worldwide ACS registries, the estimated prevalence of diabetes among ACS patients was about $20-30 \%{ }^{(\mathbf{1 9}, \mathbf{2 0})}$.
Our work showed statistical significant difference between studied groups as regard $\mathrm{T}$ Chol and LDL-C, Trg and HDL-C, between control and ACS groups, and also between SA and ACS groups in $\mathrm{T}$ Chol and HDL-C and Trg. Among ACS subgroups there were no significant differences as regarding levels of $\mathrm{T}$ Chol, triglycerides, HDL-C and LDL-C. Kazama et al. ${ }^{(17)}$, on comparing the control group versus the CHD groups for body mass index (BMI) and levels of TC, TG, and hsCRP found that there was significant increase, while serum levels of HDL-c decreased significantly ${ }^{(\mathbf{1 3})}$.

In our study, there was significant difference between the studied groups regarding AST, while there were non-significant differences in ALT, creatinine and urea level among groups. In contrast to our study was Arsalan et al. ${ }^{(21)}$, who had recorded significantly higher mean levels of creatinine and urea in control group, compared with ACS patients.

Also, regarding hsCRP, we found significant difference between the studied groups, with increased levels in ACS groups. Also we noticed that there was significant difference between US group, STEMI and non STEMI groups. Together with us were many authors from them were Wang et al. ${ }^{(18)}$, who observed that hsCRP increased significantly among ACS group, which confirms the role of inflammation in development of CAD. There was significant correlation between the plasma level of hsCRP and angiographic severity of coronary atherosclerosis as assessed by Gensini score. In patients with ACS elevated hsCRP is associated with higher rate of complication ${ }^{(12,22)}$.

Omentin-1 level, in our study, showed significant decreased level in diseased groups compared to control group. Also there was decrease in mean omentin-1 between SA and ACS but it was nonstatistical significance. Among ACS groups there were no significant differences as regarding omentin-1 levels. In agreement with us was Wong (23), who demonstrated that omentin-1 levels were lower in patients with CAD compared with controls. 
Moreover, serum omentin-1 concentrations were independently correlated with CAD. Patients with ACS also had lower serum concentrations of omentin-1 compared with patients with SA. Agasthi et al. and Motawi et al. ${ }^{(24,25)}$ concluded that serum omentin-1 level is independently and negatively associated with CAD. In contrary, were Feng and $\mathbf{X u}$ (26), who found that there were no changes in plasma omentin-1 levels in ACS group compared with stable angina pectoris group.

We found that, there was significant positive correlation between omentin-1 level and triglycerides, and statistical significant negative correlation between omentin-1 level and hsCRP among the studied groups. Among ACS subgroups, there were non- significant negative correlation between omentin1 level and BMI, T Chol, LDL-C, urea, creatinine and AST. While there were non-significant positive correlation between omentin-1 level and triglycerides, HDL-C, and ALT.

Going on with us, Zhang et al. (27) observed that severity of ACS correlates directly with deranged lipid profile like raised LDL-C, triglyceride/HDL-C ratio, T Chol and HDL-C. So, severity and incidence of ACS in diabetic patients can be minimized by maintaining adequate glycemic control and also by keeping circulating lipids under control. Omentin-1 may be protective and proinflammatory cytokines. Additionally, omentin-1 may be related to lipid metabolism ${ }^{(6,11,13)}$. But Shazia et al. (28) said "ACS has no association with LDL-C and T Chol levels".

Our study found that ROC curve analysis of; omentin- 1 at cut off $\leq 97.5 \mathrm{ng} / \mathrm{L}$ has 0.816 AUC, $83.3 \%$ sensitivity, $71.7 \%$ specificity, $59.5 \%$ PPV, 89.6\% NPV with $75.6 \%$ accuracy; and chemerin revealed an AUROC of 0.949 which was comparable with that of hsCRP (0.946). At cut off value of $>375$ $\mathrm{ng} / \mathrm{L}$, serum chemerin predicted CAD in type 2 diabetic patients with $96.7 \%$ sensitivity and $86.6 \%$ specificity. When using chemerin, omentin-1 and hsCRP in combination, all CAD were correctly diagnosed (100\% sensitivity). Yildiz et al. ${ }^{(29)}$ showed that an omentin-1 level $\leq 291 \mathrm{ng} / \mathrm{L}$ had a sensitivity of $69 \%$ and specificity of $75 \%$ in predicting adverse cardiac events in patients with hypertrophic cardiomyopathy. Stejskal et al. (30) stated that omentin-1 seems to be a useful biomarker of coronary artery disease across the whole age spectrum. Omentin-1 might be involved in the development of CAD via dysfunction of endothelium dependent coronary dilatation, insulin activity and inflammation ${ }^{(6)}$.

On studying the coronary artery disease participants on basis of diabetic parameters, we divided them into: CAD without DM type 2 and CAD with DM type 2 . There was high statistical significant difference between the groups as regarding FBG, HbA1c, T Chol, HDL-C, LDL-C, hsCRP, chemerin and omentin-1. The present study revealed that chemerin level was significantly higher in CAD with type 2 diabetic patients compared to control group and in CAD with diabetic patients compared to those without DM. Our results were confirmed by previous researches obtained by Elsaid et al. ${ }^{(31)}$ that reported increased serum chemerin in type 2 diabetic patients with atherosclerosis ${ }^{(13,15)}$. In contrast, El-Mesallamy et al. (32) didn't reveal significant differences in serum chemerin between CAD patients with and without type 2 diabetes.

Our study showed that omentin-1 level was significantly lower in CAD with type 2 diabetic patients compared to control group and in CAD with diabetic patients compared to those without DM.

Our study revealed significant positive correlation between chemerin level and hsCRP while there was significant negative correlation between omentin- 1 and both chemerin and hsCRP in CAD with and without type 2 diabetic patients. Similar findings were reported previously in metabolic syndrome ${ }^{(11,13,33,34)}$.

Logistic regression analysis revealed that increasing total cholesterol, triglycerides, LDL-C, hsCRP and chemerin. Decreasing HDL-C and omentin1 are independent predictors of CAD in type 2 diabetic patients. Confirming our results were Kaur et al. ${ }^{(7)}$ and Zhou et al. ${ }^{(35)}$.

\section{CONCLUSION}

Serum chemerin was increased, while omentin-1 is decreased in CAD associated with type 2 diabetic patients. They were correlated with disease severity and can be used in prediction of CAD in type 2 diabetic patient. Further large sample size studies are recommended to confirm these results.

\section{REFERENCES}

1. De Luca G, Bellandi F Huber K et al. (2011): Early glycoprotein IIb-IIIa inhibitors in primary angioplasty abciximab long-term results (EGYPT-ALT) cooperation: individual patient's data meta-analysis. J Thromb Haemost., 9:2361-70.

2. American Diabetes Association (2007): Standards of medical care in diabetes. Diabetes Care, 30(1): 4-41.

3. Gelsinger $\mathrm{C}$, Tschoner A, Kaser $\mathrm{S}$ et al. (2010): Adipokine update: New molecules, new functions. Wien Med Wochenschr., 160: 377-90.

4. Cheng X (2016): Elucidating the pathophysiological significance of circulating omentin levels: Is higher better? Atherosclerosis, 251: 522-524.

5. Kaushik N, Kaushik R, Dixit $P$ et al. (2018): Plasma omentin-1 level and its relationship with insulin resistance in obese prediabetics. Journal of Clinical and Diagnostic Research, 12 (4): 10-14.

6. Askin L, Duman H, Ozylldiz A et al. (2020): Association between omentin-1 and coronary artery 
disease: Pathogenesis and clinical research. Current Cardiology Reviews, 16(3) 198-201.

7. Kaur J, Adya R, Tan B et al. (2010): Identification of chemerin receptor (ChemR23) in human endothelial cells: Chemerin-induced endothelial angiogenesis. Biochem Biophys Res Comnmn., 391: 1762-1768.

8. Seldinger $\mathbf{S}$ (1953): Catheter replacement of the needle in percutaneous arteriography. Acta Radio., 139: 368-376.

9. Gensini G (1983): A more meaningful scoring system for determining the severity of coronary heart disease. Am $\mathbf{J}$ Cardiol., 5:606-9.

10. American Diabetes Association (2016): Classification and diagnosis of diabetes mellitus. Diabetes Care, 39(1): 13-22.

11. Sitar-Taut A, Coste S, Tarmure S et al. (2020): Diabetes and obesity-Cumulative or complementary effects on adipokines, inflammation, and insulin resistance. Journal of Clinical Medicine, 9(9): 2077-2383.

12. Niersmann $C$, Röhrig $K$, Blüher $M$ et al. (2020): Increased release of proinflammatory proteins in primary human adipocytes and activation of the inflammatory NFKB, p38, and ERK pathways upon omentin treatment. Obes Facts, 13:221-236.

13. Gateva A, Assyov Y, Tsakova A et al. (2018): Classical (adiponectin, leptin, resistin) and new (chemerin, vaspin, omentin) adipocytokines in patients with prediabetes. DOI: https://doi.org/10.1515/hmbci-2017-0031 .

14. Ernst M, Haidl I, Zúñiga L et al. (2011): Disruption of the chemokine-like receptor-1 (CMKLR1) gene is associated with reduced adiposity and glucose intolerance. Endocrinology, 153(2):672-682.

15. Xiang Z, Yifei T, Yuqi C et al. (2019): Serum chemerin as a novel prognostic indicator in chronic heart failure. https://www.ncbi.nlm.nih.gov/pmc/articles/PMC676165 $8 /$

16. Zhong $X$, Zhang $H$, Tan $H$ et al. (2011): Association of serum omentin-1 levels with coronary artery disease. Acta Pharmacol Sin., 32(7): 873-878.

17. Kazama K, Usui T, Okada M et al. (2012): Omentin plays an anti- inflammatory role through inhibition of TNF-alpha-induced superoxide production in vascular smooth muscle cells. Eur J Pharmacol., 686(1-3): 116-123.

18. Wang X, Ouyang Y, Liu J et al. (2014): Fruit and vegetable consumption and mortality from all causes, cardiovascular disease, and cancer: systematic review and dose-response meta- analysis of prospective cohort studies. BMJ., 349: 4490-6.

19. Peterson E, Roe M, Chen A et al. (2010): The NCDR ACTION Registry-GWTG: transforming contemporary acute myocardial infarction clinical care. Heart, 96(22):1798-802.

20. Muhammad K, Umar Y, Susan E et al. (2017): Prevalence of undiagnosed type 2 diabetes in patients admitted with acute coronary syndrome: the utility of easily reproducible screening methods. BMC Endocrine Disorders BMC series. Obesity (Silver Spring), 19: 1552-1559.

21. Arsalan $M$, Syed $A$, Aleena $Z$ et al. (2018): Efficacy of serum blood urea nitrogen, creatinine and electrolytes in the diagnosis and mortality risk assessment of patients with acute coronary syndrome. Indian Heart J., 70(2018) 353-359.

22. Masood A, Jafar S, Akram Z (2011): Serum high sensitivity C-reactive protein levels and the severity of coronary atherosclerosis assessed by angiographic Gensini score. Journal of the Pakistan Medical Association, 61(4):325-7.

23. Wong N (2014): Epidemiological studies of CHD and the evolution of preventive cardiology. Nature Reviews. Cardiology, 11 (5): 276-89.

24. Agasthi P, Aloor S, Axiyan $M$ et al. (2015): Association between serum omentin-1 level and coronary artery disease: A meta- analysis. Arteriosclerosis, Thrombosis, and Vascular Biology, 35: A548.

25. Motawi M, Soliman G, Maha M et al. (2018): Serum levels of chemerin, apelin, vaspin, and omentin-1 in obese type 2 diabetic Egyptian patients with coronary artery stenosis. Can J Physiol Pharmacol., 96: 38-44.

26. Feng $X$ and $X u Y$ (2009): Relationship between plasma P-selectin and coronary heart disease. Chin J Postgraduates Med., 32:34-8.

27. Zhang M, Xinrui T, Chunyan Y et al. (2017): Serum levels of omentin-1 are increased after weight loss and are particularly associated with increases in obese children with metabolic syndrome. Foundation Acta Pædiatrica, 106: 1851-1856.

28. Shazia N, Sheeba F, Uzma T (2017): Association of serum omentin-1 levels with coronary artery disease (CAD) in a local population of Karachi A multicenter study. ASH and KMDC., 22(1):31-36.

29. Yildiz S, Sahin I, Cetinkal G et al. (2018): Usefulness of serum omentin-1 levels for the prediction of adverse cardiac events in patients with hypertrophic cardiomyopathy. Med Princ Pract., 27:107-114.

30. Stejskal D, Vaclavik J, Smekal A et al. (2016): Omentin-1 levels in patients with premature coronary artery disease, metabolic syndrome and healthy controls: Short communication. Biomed Pap Med Fac Univ Palacky Olomouc Czech Repub., 160(2):219221.

31. Elsaid N, Fawzy M, Mohammed N et al. (2013): Role of serum chemerin level in atherosclerosis in Egyptian patients with type 2 diabetes mellitus. Journal of applied Sciences Research, 9(1):778-785.

32. El-Mesallamy H, El-Derany M, Hamdy N (2011): Serum omentin- 1 and chemerin levels are interrelated in patients with Type 2 diabetes mellitus with or without ischaemic heart disease. Diabetic Medicine, 28(10):11941200.

33. Abdelhaleem A, Al-salamony A (2012): Serum chemerin and adiponectin levels in metabolic syndrome patients with or without coronary artery diseases. Journal of American Science, 8(9): 387-394.

34. Lehrke M, Becker A, Greif M (2009): Chemerin is associated with markers of inflammation and components of the metabolic syndrome but does not predict coronary atherosclerosis. Eur J Endocrinol., 161: 339-344.

35. Zhou J, Tong $X$, Zhu $\mathrm{L}$ et al. (2017): Plasma omentin-1 level as a predictor of good coronary collateral circulation. J Atheroscler Thromb., 24(9):940-948. 